\title{
From Equilibrium to Steady State: The Transient Dynamics of Colloidal Liquids under Shear
}

\author{
J Zausch $^{1}$, J Horbach ${ }^{1,2}$, M Laurati ${ }^{3}$, S U Egelhaaf ${ }^{3}$, J M \\ Brader $^{4}$, Th Voigtmann ${ }^{2,4}$, and M Fuchs ${ }^{4}$ \\ ${ }^{1}$ Institut für Physik, Johannes-Gutenberg-Universität Mainz, Staudinger Weg 7, \\ 55099 Mainz, Germany \\ ${ }^{2}$ Institut für Materialphysik im Weltraum, Deutsches Zentrum für Luft- und \\ Raumfahrt (DLR), 51170 Köln, Germany \\ ${ }^{3}$ Condensed Matter Physics Laboratory, Heinrich-Heine-Universität, \\ Universitätstraße 1, 40225 Düsseldorf, Germany \\ ${ }^{4}$ Fachbereich Physik, Universität Konstanz, 78457 Konstanz, Germany
}

\begin{abstract}
We investigate stresses and particle motion during the start up of flow in a colloidal dispersion close to arrest into a glassy state. A combination of molecular dynamics simulation, mode coupling theory and confocal microscopy experiment is used to investigate the origins of the widely observed stress overshoot and (previously not reported) super-diffusive motion in the transient dynamics. A link between the macro-rheological stress versus strain curves and the microscopic particle motion is established. Negative correlations in the transient auto-correlation function of the potential stresses are found responsible for both phenomena, and arise even for homogeneous flows and almost Gaussian particle displacements.
\end{abstract}

\section{Introduction}

Relaxation processes in highly viscous liquids are strongly affected by the application of an external shear field. Even at small shear rates, the shear viscosity can decrease by orders of magnitude while the structure exhibits only small changes compared to equilibrium. This phenomenon of shear thinning has been observed in experiments of various systems, such as colloids and polymers, as well as in computer simulations of model systems [1]. The transport coefficients measured in quiescent equilibrium or linear response markedly differ from those in steady-shear states. This raises the question how the system's microscopic dynamics evolves in response to a sudden change in the externally applied shear. This concerns the transient dynamics, as, for example, displayed in time-dependent transport coefficients or waiting-time dependent two-time correlation functions. Here, we are concerned with a suddenly commencing shear flow, imposed upon a quiescent, equilibrated system. While the measurement of macroscopic quantities like the shear stress $\sigma$ is standard rheology procedure, the detailed study of microscopic dynamics has been a recent contribution to the field from both computer simulation [2, 3, 4, [5, 6, 7, 8, 9] and direct-imaging techniques for colloidal suspensions, namely confocal microscopy [10].

It is well known that after starting a steady shear flow, stresses develop in the liquid. At short times after switching on (corresponding to small overall strains $\gamma=\dot{\gamma} t$ ), they increase almost linearly and at long times saturate at a constant level, the 
stress corresponding to the sheared steady state. In many cases, the increase in stress first continues beyond this steady-state value before relaxing back at larger times. A nonmonotonic $\sigma(t)$-versus- $t$ curve results, with a maximum at intermediate strains, the 'stress overshoot'. Such overshoots have been seen in polymeric liquids [11, 12, 13], metallic glasses [14, 15], and in simulations [6, 16, 17, 18, 19]. Despite its abundance among different systems, no generally accepted physical picture has emerged. Linking this macroscopically observed feature to the underlying microscopic dynamics can be achieved by studying the transient (microscopic) correlation functions. We pursue this by combining confocal microscopy, molecular-dynamics simulation, and a modecoupling-theory approach. Surprisingly, we find that a previously not reported, superdiffusive particle motion is characteristic for the dynamics at strain values where the stress overshoot appears. We connect both phenomena to negative portions in the transient auto-correlation function of the microscopic stresses.

The mode-coupling theory (MCT) for colloidal rheology 20, 21, 22, 23, 24] is a recent microscopic framework from which constitutive equations can be derived that capture the interplay of slow dynamics and shear thinning. The theory can thus address the above question on the relation between microscopic dynamics and macroscopic rheology. Its underlying physical picture is a combination of the cage effect, leading to slow dynamics, and of the advection of long-wavelength fluctuations to short wavelength, leading to a shear-induced breaking of cages [25, 26, 27, 28]. The $\mathrm{MCT}$ equations can be formulated from an integration-through-transient formalism developed by Fuchs and Cates [20, 21]. In this formalism, transient correlation functions of a special kind (equilibrium averages over fluctuations that evolve according to the nonequilibrium dynamics) play a central rôle. The direct measurement of such correlations, which is possible under shear startup, provides a direct link between theory, simulation and experiment. This allows for a detailed test of some of the assumptions implicit in the theory.

The paper is structured as follows: we will in section 2 discuss the different techniques used in this study, viz. the simulation model, experimental setup, and theoretical framework. Section 3 presents and compares the main results for both stress buildup and waiting-time dependent mean-squared displacements after switching on shear flow. Finally, section 4 concludes.

\section{Methods and Techniques}

\subsection{Simulation model and details of the simulation}

Molecular dynamics computer simulations have been done for a simple model of a binary $\mathrm{AB}$ mixture of charged colloids. The interactions between the particles are modeled by a Yukawa potential,

$$
u_{\alpha \beta}=\epsilon_{\alpha \beta} d_{\alpha \beta} \frac{\exp \left(-\kappa_{\alpha \beta}\left(r-d_{\alpha \beta}\right)\right)}{r} \quad \alpha, \beta=\mathrm{A}, \mathrm{B},
$$

which is truncated at a cut-off distance $r_{\mathrm{c}}^{\alpha \beta}$, defined by $u_{\alpha \beta}\left(r_{\mathrm{c}}^{\alpha \beta}\right)=10^{-7} \epsilon_{\mathrm{AA}}$. The "particle diameters" are set to $d \equiv d_{\mathrm{AA}}=1.0, d_{\mathrm{BB}}=1.2 d$ and $d_{\mathrm{AB}}=1.1 d$, the energy parameters to $\epsilon \equiv \epsilon_{\mathrm{AA}}=1.0, \epsilon_{\mathrm{BB}}=2.0 \epsilon, \epsilon_{\mathrm{AB}}=1.4 \epsilon$, and the screening parameters to $\kappa_{\mathrm{AA}}=\kappa_{\mathrm{BB}}=\kappa_{\mathrm{AB}}=6 / d$. The choice of these parameters ensures that, at the density $\varrho=0.675 \mathrm{~m}_{\mathrm{A}} / d_{\mathrm{AA}}^{3}$ considered in this work, no problems with crystallization or phase-separation occur, at least in the temperature range under consideration. The masses of the particles are set to unity, i.e. $m=m_{\mathrm{A}}=m_{\mathrm{B}}=1.0$. 
The simulations were done for a 50:50 mixture of $N=2 N_{\mathrm{A}}=2 N_{\mathrm{B}}=1600$ particles, placed in a cubic simulation box of linear size $L=13.3 d$. For the sheared system, we chose the $x$ direction as the direction of shear and the $y$ and $z$ direction as the gradient and vorticity direction, respectively. Shear was imposed onto the system via modified periodic boundary conditions, the so-called Lees-Edwards boundary conditions [29, 30]. Here, a particle that moves out of the simulation box in $y$ direction is subject to a displacement in $x$ direction due to constant velocities $u_{\mathrm{s}, x}$ and $-u_{\mathrm{s}, x}$ of the image cells above and below the simulation cell, respectively. For the glassforming Yukawa system considered in this work, the application of Lees-Edwards boundary conditions leads to a linear shear profile in the steady state regime, $v_{\mathrm{s}, x}(y)=\dot{\gamma}(y-L / 2)$ with the shear rate $\dot{\gamma}=u_{\mathrm{s}, x} / L$. In recent simulation studies, Lees-Edwards boundary conditions have been used in conjunction with the so-called Sllod equations of motion [30] to enforce the formation of a linear shear profile. For our purpose, the use of the Sllod equations is not appropriate since we are mainly interested in the study of the transient dynamics, i.e. in the time regime before the steady state regime is reached. In this case, one may expect that the emergence of non-linear shear profiles strongly affects the response of the system to the external shear field. Thus, the Sllod equations would modify the transient dynamics in an artificial manner. However, as we shall see below, an almost linear profile is built up long before the steady state regime is approached. So our simulations indicate a posteriori that it does not matter whether or not the Sllod equations are used. We also note that non-linear shear profiles may occur as a consequence of a hydrodynamic instability, leading to the formation of shear bands. However, in the glassforming Yukawa system of this work, such phenomena are not observed, at least for the considered range of shear rates.

Both for the equilibrium simulations and the simulations under shear, the system was coupled to a dissipative particle dynamics (DPD) thermostat (see Ref. 31] and references therein). The DPD equations of motion are given by

$$
\dot{\mathbf{r}}_{i}=\frac{\mathbf{p}_{i}}{m_{i}}, \quad \dot{\mathbf{p}_{i}}=\sum_{j(\neq i)}\left[\mathbf{F}_{i j}+\mathbf{F}_{i j}^{\mathrm{D}}+\mathbf{F}_{i j}^{\mathrm{R}}\right]
$$

with $\mathbf{r}_{i}$ and $\mathbf{p}_{i}$ the position and momenta of a particle $i(i=1, \ldots, N)$. In Eq. (2) $\mathbf{F}_{i j}=-\nabla u_{i j}$ denotes the conservative force between a particle $i$ and a particle $j$ due to the interaction potential defined by Eq. (1). To provide the thermostatting of the system a dissipative force $\mathbf{F}_{i j}^{\mathrm{D}}$ and a random force $\mathbf{F}_{i j}^{\mathrm{R}}$ are added in Eq. (2).

The dissipative force is defined by 32

$$
\mathbf{F}_{i j}^{\mathrm{D}}=-\zeta w^{2}\left(r_{i j}\right)\left(\hat{\mathbf{r}}_{i j} \cdot \mathbf{v}_{i j}\right) \hat{\mathbf{r}}_{i j}
$$

with $\zeta$ being a friction coefficient, $\mathbf{v}_{i j}=\mathbf{v}_{i}-\mathbf{v}_{j}$ the relative velocity between particle $i$ and $j, \hat{\mathbf{r}}_{i j}$ the unit vector of the vector $\mathbf{r}_{i j}=\mathbf{r}_{i}-\mathbf{r}_{j}$, and $r_{i j}=\left|\mathbf{r}_{i}-\mathbf{r}_{j}\right|$ the distance between particle $i$ and $j$. The function $w\left(r_{i j}\right)$ is defined by $w\left(r_{i j}\right)=\sqrt{1-r_{i j} / r_{c}}$ for $r_{i j}<r_{c}^{\mathrm{DPD}}=1.25 d$ and $w\left(r_{i j}\right)=0$ otherwise. Thus, the force $\mathbf{F}^{\mathrm{D}}$ describes a frictional force due to the interaction between neighboring particle pairs. The use of the relative velocities between neighboring particles in (3) is crucial to obtain the correct behaviour on hydrodynamic scales. It ensures that the DPD thermostat is Galilean invariant and that momentum is locally conserved. For the friction coefficient $\zeta$ we chose the value $\zeta=12$. With this value, the microscopic properties are close to those of a purely Newtonian dynamics with $\zeta=0$. The microscopic dynamics can be significantly changed by using high values of $\zeta$, approaching the limit of an overdamped stochastic dynamics where inertia effects can be effectively neglected. In 
a forthcoming publication [33], we present simulations with $\zeta=1200$ and compare them to those with $\zeta=12$ that are shown in the present paper. These simulations indicate that qualitatively all the essential features of the transient dynamics do not depend on the choice of $\zeta$.

The random force in (2) is given by $\mathbf{F}_{i j}^{\mathrm{R}}=\sqrt{2 k_{B} T \zeta} w\left(r_{i j}\right) \theta_{i j} \hat{\mathbf{r}}_{i j}$ where $\theta_{i j}=\theta_{j i}$ are uniform random numbers with zero mean and unit variance. The amplitude of the random force, $\sqrt{2 k_{B} T \zeta}$, is chosen in accordance with the fluctuation-dissipation theorem.

The equations of motion were integrated by a generalized form of the velocity Verlet algorithm that has been recently proposed by Peters [34]. For the time step of the integration we used $\delta t=0.0083 \tau$ (with the time unit $\tau=\sqrt{m d^{2} / \epsilon}$ ). First, the samples were fully equilibrated in the temperature range $1.0 \geq T \geq 0.14$, performing at least 30 independent runs at each temperature. Starting from fully equilibrated samples, production runs at various shear rates were performed. These runs were sufficiently long to reach the steady state regime.

At the lowest temperature $T=0.14,250$ independent runs were done, each of them over 40 million time steps. This relatively large effort was necessary for an accurate determination of the shear stress, defined by

$$
\left\langle\sigma_{x y}\right\rangle=\frac{1}{L^{3}}\left\langle\sum_{i}\left[m_{i}\left(v_{i, x}-v_{\mathrm{s}, x}(y)\right) v_{i, y}+\sum_{j>i} r_{i j, x} F_{i j, y}\right]\right\rangle .
$$

The shear stress $\left\langle\sigma_{x y}\right\rangle$ is a collective quantity and thus lacks the self-averaging property of one-particle quantities. Therefore, a relatively large number of independent runs have to be made to obtain $\left\langle\sigma_{x y}\right\rangle$ with a reasonable accuracy. Below we also present simulation results for the mean squared displacement (MSD) of a tagged particle,

$$
\left\langle r^{2}(t)\right\rangle=\left\langle\left(r_{\text {tag }, \alpha}(t)-r_{\text {tag }, \alpha}(0)\right)^{2}\right\rangle, \quad \alpha=x, y, z,
$$

where $r_{\mathrm{tag}, \alpha}(t)$ is the $\alpha$ component of the position of the tagged particle at time $t$ (an average is performed over all particles of the same species). The MSD is an example of a one-particle quantity. From the MSD, the self-diffusion constant $D$ can be calculated via the Einstein relation,

$$
D=\lim _{t \rightarrow \infty} \frac{\left\langle r^{2}(t)\right\rangle}{2 t} .
$$

Note that for the sheared system, the MSD as well as the self-diffusion constants are anisotropic and depend on the considered Cartesian direction (see below).

Another quantity, that we use to characterize the difference between sheared and unsheared systems, is the so-called non-Gaussian parameter [35, 36],

$$
\alpha_{2}(t)=\frac{1\left\langle r^{4}(t)\right\rangle}{3\left\langle r^{2}(t)\right\rangle^{2}}-1
$$

$\alpha_{2}(t)$ is the coefficient of first correction term to the Gaussian approximation of the incoherent intermediate scattering function. Note that $\alpha_{2}(t)$ vanishes in the diffusive long-time limit when the Einstein relation (6) holds.

\subsection{Experimental techniques}

2.2.1. Samples We used polymethylmethacrylate (PMMA) colloids fluorescently labeled with nitrobenzoxadiazole (NBD) and dispersed in a mixture of cycloheptyl 
bromide and cis-decalin that closely matches the density and refractive index of the colloids. Since the colloids acquire a small charge in this solvent mixture, we added $4 \mathrm{mM}$ of tetrabutylammonumchloride in order to screen the charges [37. Such a system shows nearly hard-sphere (HS) behaviour, where the volume fraction $\phi=(\pi / 6) n d^{3}$ is the only thermodynamic control parameter.

The particle diameter $d=1680 \pm 4 \mathrm{~nm}$ was determined by static light scattering on a very dilute colloidal suspension $\left(\phi \simeq 10^{-4}\right)$. The diameter was also independently estimated from the position of the first peak of the radial distribution function obtained by confocal microscopy [38, 39], yielding $d=1690 \mathrm{~nm}$. With the very dilute sample we also performed dynamic light scattering. This confirmed the determined particle diameter and, in addition, allowed us to deduce the relative polydispersity in size, 0.062 , from the angular dependence of the diffusion coefficient [40].

The colloid volume fraction of the stock solution was calibrated by drying. A drop of the suspension was weighed and allowed to dry in a vacuum oven until all the solvent was evaporated. The weight fraction was then calculated as $\phi_{w}=m_{\mathrm{dry}} / m_{\mathrm{total}}$, where $m_{\text {dry }}$ is the mass of the dried sample and $m_{\text {total }}$ the original mass before drying. The colloid volume fraction was estimated from the weight fraction as $\phi=\alpha \phi_{w}$, where the factor $\alpha$ takes into account the contribution of the PHSA hairs, which are collapsed in the dried state. Following [41] we assume $\alpha=1.04$. Our sample was prepared at $\phi=0.57$. The volume fraction of the sample was also determined by direct imaging and found to be consistent.

2.2.2. Shear Cell Shear was applied to the sample by means of a shear cell designed and built in our lab. It represents an improved version of cells previously used in light scattering and microscopy experiments [42, 43, 44]. For the present microscopy experiments, a specially designed microscope stage is used to mount the shear cell in the microscope. The cell consists of two parallel glass coverslips (thickness $170 \mu \mathrm{m}$ ) whose separation can be continuously varied within the range $300 \ldots 1000 \mu \mathrm{m}$ by vertically shifting the upper plate, which is sitting on three metal spheres. Adjusting the position of the metal spheres also ensures that the two plates are parallel; this is verified as described before [45]. In the present experiment we used a gap width $(320 \mu \mathrm{m})$ close to the minimum value in order to achieve maximum strain. The bottom plate of the cell is driven by a piezoelectric actuator (PI Instruments, model P-841.60) which can provide a maximum stroke of $90 \mu \mathrm{m}$. The other end of the bottom plate is connected to a pivoted lever driving the upper plate in the direction opposite to the bottom plate [42]. The vertical position of the pivot moves the vertical position of the stationary plane inside the sample and changes the maximum displacement of the top plate. In this experiment the maximum displacement of the top plate was $630 \mu \mathrm{m}$, as measured by a displacement sensor (Lion Precision, model ECL100-U8A, displacement range $2 \mathrm{~mm}$ ) which was calibrated prior to the measurement using the piezoelectric actuator displacement, which is known with nanometer accuracy. With this arrangement the maximum obtainable strain is $\gamma=2.25$.

A constant shear rate is achieved by driving the piezoactuator with a linear voltage ramp which covers the whole displacement range. The slope of the ramp determines the shear rate $\dot{\gamma}$. The shear rate is also experimentally measured from the velocity profile across the gap, as explained below.

In order to minimize solvent evaporation during the experiments, the two glass plates are surrounded by teflon sheets which touch each other and thus seal the sample environment. Due to the very low friction of teflon, no change in the plate displacement 


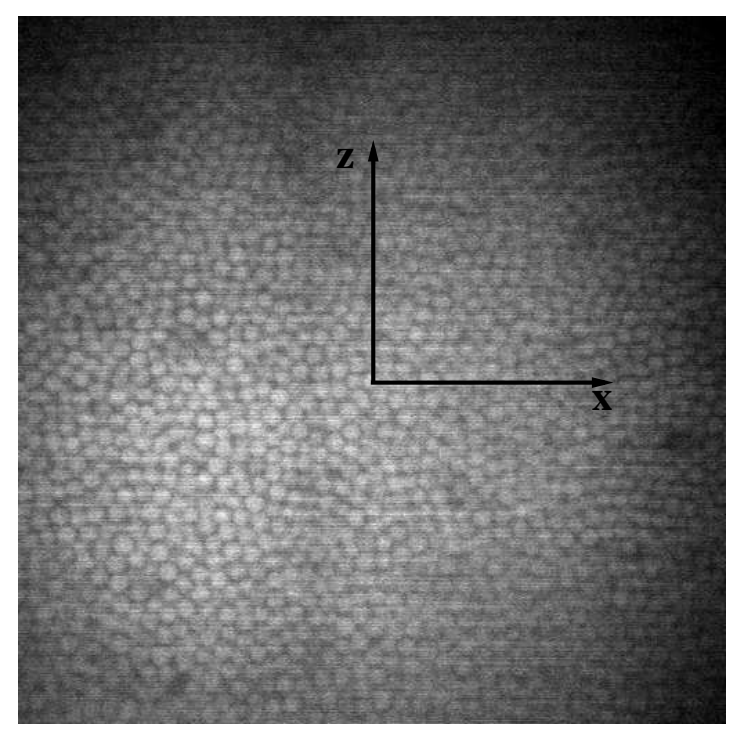

Figure 1. Confocal microscopy image of the sample at switch on of shear. The axes $x$ and $z$ indicate respectively the velocity and vorticity direction. The gradient direction is perpendicular to the plane of the image.

due to the contact of the teflon sheets is observed, as verified during calibration.

In recent investigations of the flow of glassy colloidal dispersions wall slip was observed when smooth surfaces were used to apply shear to the samples. It was shown [10] that slip can be prevented when the surfaces are coated with colloidal particles similar to those used for sample preparation. Although our volume fraction is below the glass transition, we coated the coverslips with a layer of polydisperse colloids. The velocity profile across the gap indeed shows no indication of wall slip (see below).

2.2.3. Confocal Microscopy and Image Analysis Confocal Microscopy experiments were performed with a fast-scanning VT-Eye confocal microscope (Visitech International) mounted on a Nikon TE2000-U inverted microscope. A Nikon Plan Apo VC $100 \times$ oil immersion objective was used for all measurements. Two-dimensional images of the samples were recorded at a depth of $15 \mu \mathrm{m}$ inside the sample (except for the determination of the velocity profile, see below), in order to avoid boundary effects and to retain good quality images. Images have $512 \times 512$ pixels, corresponding to an area of $57 \times 57 \mu \mathrm{m}^{2}$.

In a typical experiment 500 images were recorded starting simultaneously with the application of shear and ending when the maximum strain was reached. Image acquisition and application of shear were synchronized by starting the voltage ramp, which controls the displacement of the plates, with a trigger provided by the confocal microscope. The response of the piezoactuator to the voltage ( $\mu$ s delay) can be considered instantaneous on the time scale of our experiments.

Figure 1 shows a typical confocal image acquired in experiments. The axes $x$ and $z$ indicate the velocity and vorticity direction. The gradient direction, $y$, is perpendicular to the plane of the image. Particle coordinates and particle trajectories were extracted from images using standard routines [46]. The shear rate applied here 


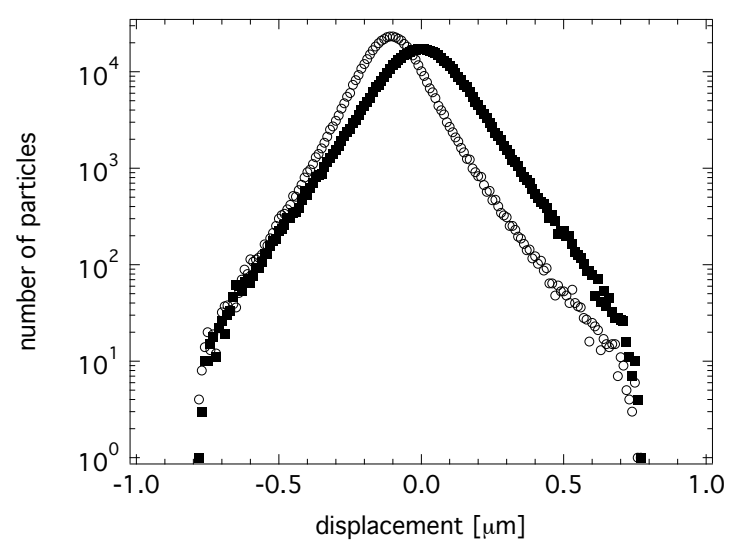

Figure 2. Histogram of particle displacements between consecutive images along the velocity $x(0)$ and vorticity $z(\mathbf{\square})$ direction.

$\left(\dot{\gamma}=0.045 \mathrm{~s}^{-1}\right)$ is sufficiently small so that particles do not move very far between two consecutive frames even in the $x$ direction. It is thus not necessary to remove the affine motions before tracking [47]. This is supported by the histograms of the frame by frame displacements in $x$ and $z$ directions as determined during a step rate experiment (Fig. 2); both histograms lie well within the displacement axis, which covers the maximum detectable range (about $\pm 1 \mu \mathrm{m}$ ).

Particle trajectories were used to calculate mean-squared displacements (MSD) and non-Gaussian parameters $\left(\alpha_{2}\right)$ in the vorticity direction $z$, according to equations 5 and 7 respectively. Only trajectories starting at commencement of shear are included in the MSD and $\alpha_{2}$ calculations in order not to loose information on the time dependence of the MSD and $\alpha_{2}$ after switching on the shear field. This implies that in each experiment only a limited number of particles are contributing to the average value of the two quantities; initially of the order of 1000 and the number decreasing with time as particles leave the field of view due to motion in or out of the observation plane. In order to improve statistics, MSDs and $\alpha_{2}$ extracted from several, typically 20, experiments were averaged after checking reproducibility.

The velocity profile across the gap was determined immediately after the application of shear by recording image series at different depths into the sample. The depth was increased in steps of $10 \mu \mathrm{m}$, from the first layer of particles above the coating up to $100 \mu \mathrm{m}$ into the sample. Deeper in the sample image quality did not allow for a quantitative analysis. Displacements between consecutive images were determined by maximizing the correlation between consecutive images [47]. The obtained velocity profile (Fig. 9) shows a linear dependence with a shear rate $\dot{\gamma}=0.0458 \mathrm{~s}^{-1}$, in good agreement with the nominal value (for a detailed discussion see below).

\subsection{Mode coupling theory for glassforming liquids under shear}

Recent advances 20, 21, 23, 24] have generalized the mode coupling theory of the glass transition to describe dense colloidal suspensions in a flowing solvent, characterized by a non-vanishing velocity gradient tensor of arbitrary time dependence $\boldsymbol{\kappa}(t)$. The resulting non-linear theory provides a route to calculating non-equilibrium averages of functions of the particle coordinates. In particular, a closed microscopic expression 
for the shear-stress in terms of the full flow history of the system, i.e., a constitutive equation relating stress and strain, may be obtained. The starting point of the theory is the many-body Smoluchowski equation [48]

$$
\partial_{t} \Psi(t)=\Omega(t) \Psi(t) \equiv \sum_{i} \boldsymbol{\partial}_{i} \cdot\left[D_{0}\left(\boldsymbol{\partial}_{i}-\beta \boldsymbol{F}_{i}\right)-\boldsymbol{\kappa}(t) \cdot \boldsymbol{r}_{i}\right] \Psi(t),
$$

where $D_{0}$ is the bare diffusion coefficient and $\beta=1 / k_{B} T$. Equation (8) describes the evolution of the probability distribution function of particle positions $\Psi(t)$ in the overdamped limit. This formulation of the theory omits hydrodynamic interactions and assumes a prescribed, spatially constant $\boldsymbol{\kappa}(t)$, thus excluding confinement effects and inhomogeneous flows such as shear-banded and shear-localized states. Although the assumption of translationally invariant flow is clearly an idealization, the simulation and experimental results presented in this work suggest that Eq. (8) is a reasonable starting point, at least for dense fluid states under shear flow. In the case of a constant shear flow $\dot{\gamma}$ along the $x$-direction and with gradient in the $y$-direction the velocity gradient tensor is given by $\kappa_{i j}=\dot{\gamma} \delta_{i x} \delta_{j u}$.

The integration through transients formalism [20, 21, 23, 24] provides a formal solution to Eq. (8) by integrating over the entire flow history. In the case of shear, the following formal result is obtained for the time-dependent distribution function

$$
\Psi(t)=\Psi_{e}+\int_{-\infty}^{t} d t^{\prime} \dot{\gamma}\left(t^{\prime}\right) \Psi_{e} \hat{\sigma}_{x y} \mathrm{e}_{-}^{\int_{t^{\prime}}^{t} d s \Omega^{\dagger}(s)},
$$

where $\hat{\sigma}_{x y}=-\sum_{i} F_{i x} r_{i y}$ is the potential part of the microscopic stress tensor and $\Omega^{\dagger}$ is the adjoint Smoluchowski operator. The time-ordered exponential function e_ arises because $\Omega^{\dagger}(t)$ does not commute with itself for different times. The assumption of an equilibrium Boltzmann distribution $\Psi_{e}$ in the infinite past allows general nonequilibrium averages to be expressed in terms of averages taken with the equilibrium distribution function. Equation (9) is an operator expression, to be used with the understanding that quantities to be averaged are placed to the right before integration over particle coordinates. Within linear response the macroscopic shear stress $\sigma(t)$ is given in terms of the shear modulus by the familiar Green-Kubo formula of equilibrium statistical mechanics. Using (9) to calculate the average of the potential part of the macroscopic stress, viz. $\hat{\sigma}_{x y} / V$, where $V$ is the system volume, yields a non-linear generalized Green-Kubo relation [20, 21, 23, 24]

$$
\sigma_{x y}(t)=\frac{1}{V} \int_{-\infty}^{t} \dot{\gamma}\left(t^{\prime}\right)\left\langle\hat{\sigma}_{x y} \mathrm{e}_{-}^{\int_{t^{\prime}}^{t} d s \Omega^{\dagger}(s)} \hat{\sigma}_{x y}\right\rangle
$$

where $\langle\cdot\rangle$ represents an average over the equilibrium distribution. Note that the flow history $\dot{\gamma}\left(t^{\prime}\right)$ appears both explicitly in Eq. (10) (recovering linear response) and implicitly (nonlinearly) through the time-evolution of the stress-stress correlation function. In the present situation of start-up shear, $\dot{\gamma}\left(t^{\prime}\right)$ vanishes for all $t^{\prime}<0$, so that the integration is only performed for $t^{\prime}>0$, where the Smoluchowski operator has no explicit time dependence. Defining the generalized dynamical shear modulus by $G(t)=\left\langle\hat{\sigma}_{x y}(t) \hat{\sigma}_{x y}(0)\right\rangle / V$ as a non-linear function of $\dot{\gamma}$, we have

$$
\sigma_{x y}(t)=\dot{\gamma} \int_{0}^{t} d t^{\prime} G\left(t^{\prime}\right)
$$

Within the mode-coupling approach $G(t)$ is approximated by projecting the dynamics onto density-pair modes corresponding to all possible wave-vector pairs and directions. 
The considerable numerical complexity of the resulting equation can be significantly reduced by employing the following isotropic approximation for the modulus [22],

$$
G(t)=\frac{k_{B} T}{60 \pi^{2}} \int d k \frac{k^{5}}{k(t)} \frac{S_{k}^{\prime} S_{k(t)}^{\prime}}{S_{k}^{2}} \Phi_{k(t)}^{2}(t),
$$

where $S_{k}$ is the static structure factor. The affine solvent flow enters via the time-dependent wavevector $k(t)$ describing the advection of density fluctuations for wave number $k$ to smaller wavelengths. The exact anisotropic advection $k(t)=$ $\sqrt{k^{2}+2 k_{x} k_{y} \dot{\gamma} t+k_{x}^{2} \dot{\gamma}^{2} t^{2}}$ needs to be approximated by an isotropic wavevector in the mode-coupling $k$-integrals, $k(t) \approx k \sqrt{1+\left(\dot{\gamma} t / \gamma_{c}\right)^{2} / 3}$, in order to reduce the computational resources required. Pre-averaging over spatial directions introduces an additional source of error when compared to full solution of the anisotropic MCT equations. To first order, we expect, this discrepancy can be compensated by introducing a characteristic strain parameter, $\gamma_{c}$, to which we assign the value $\gamma_{c}=0.1$. Evaluation of (12) requires the transient intermediate density correlator, defined by $\Phi_{k}(t)=\left\langle\varrho_{\boldsymbol{k}}^{*} \exp \left[\Omega^{\dagger} t\right] \varrho_{\boldsymbol{k}(-t)}\right\rangle / N S_{\boldsymbol{k}}$. The appearance of an advected wavevector in this definition removes the trivial decorrelation of density fluctuations arising from purely affine flow. As a consequence of the equilibrium averaging, $\Phi_{k}(t)$ only contains information regarding the strain accumulated between $t=0$ and later time $t$. MoriZwanzig type projection operator manipulations yield an equation of motion for the correlator [22]

$$
\frac{\partial}{\partial t} \Phi(t)+\Gamma_{q}\left(\Phi_{q}(t)+\int_{0}^{t} d t^{\prime} m_{q}\left(t-t^{\prime}\right) \frac{\partial}{\partial t^{\prime}} \Phi_{q}\left(t^{\prime}\right)\right)=0
$$

where $\Gamma_{q}=q^{2} D_{0} / S_{q}$ is the initial decay rate. Mode-coupling approximations provide an explicit form for the memory function

$$
m_{q}(t)=\int d \mathbf{k} \frac{n S_{q} S_{k} S_{p}}{16 \pi^{3} q^{4}}\left[\mathbf{q} \cdot \mathbf{k} c_{k(t)}+\mathbf{q} \cdot \mathbf{p} c_{p(t)}\right]\left[\mathbf{q} \cdot \mathbf{k} c_{k}+\mathbf{q} \cdot \mathbf{p} c_{p}\right],
$$

where $n$ is the number density and $c_{q}$ is the equilibrium direct correlation function, $n c_{q}=1-1 / S_{q}$. It should be noted that the colloid-colloid interaction potential and thermodynamic statepoint enter purely via the equilbrium structure factor. In the absence of flow the two vertices in (12) or (14) form a perfect square. For finite shear rates wavevector advection leads to a de-phasing of the vertices, resulting in a reduction of memory effects. This is the mechanism by which the competition between slow structural relaxation and flow enter the theory. Equations (12)-(14) form a closed theory for the calculation of $G(t)$. The corresponding shear stress follows from (11).

For the purposes of this study we consider a one-component system of hardspheres of diameter $d$, and all numerical calculations will be performed using the Percus-Yevick approximation for $S_{q}$; discretization of the integrals is performed like in Refs. 22, 49]. This minimal model provides both a reasonable first approximation to the PMMA colloids used in our experiments and captures the fundamental excluded volume effects responsible for the phenomenology observed in our binary mixture simulations. The relevant parameters in the hard-sphere system are the bare Péclet number $\mathrm{Pe}_{0}=\dot{\gamma} d^{2} / D_{0}$, measuring the importance of shear relative to Brownian motion, and the packing fraction $\phi$ which determines the timescale of structural relaxation in equilibrium. The effect of shear on the structural relaxation is measured by the dressed Péclet or Weissenberg number Pe, which we define by $\mathrm{Pe}=\dot{\gamma} d^{2} /(2 D)$, using $D$, the long time self diffusion coefficient in equilibrium. It compares the shear 


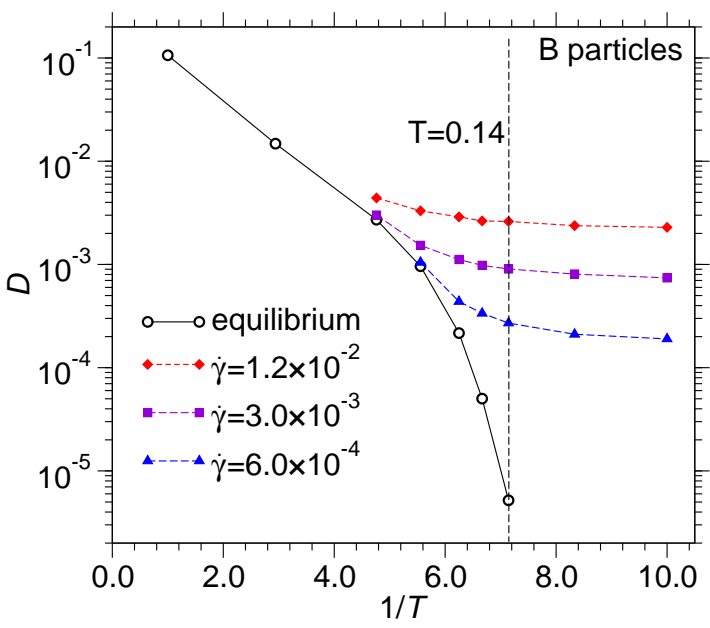

Figure 3. Self-diffusion constants for the B particles in equilibrium and at the indicated shear rates, as obtained from the simulation. For the sheared systems the diffusion coefficients are calculated from the mean squared displacement in $x$ and $y$ direction, i.e. perpendicular to the shear direction. The temperature $T=0.14$ is marked.

rate with the time required for a particle to diffuse a distance of its diameter in one spatial direction.

\section{Results}

Figure 3 shows the diffusion coefficients $D(\dot{\gamma}, T)$ obtained from the simulations of the binary Yukawa mixture at various temperatures and shear rates. Only the diffusivity of the larger particles is shown, as the smaller particles behave qualitatively identically. Open circles represent values measured in the quiescent equilibrium state, $D(\dot{\gamma}=0, T)$. They show a strong faster-than-Arrhenius slowing down with decreasing temperature, signifying that the system approaches a glass transition. On the timescale of the simulation, no diffusive regime is reached in the MSD for temperatures lower than $T=0.14$.

Applying steady shear to the system, one finds instead that even at the lowest temperatures considered here, the system shows a finite diffusivity. For a fixed value of $\dot{\gamma}$, the $D(\dot{\gamma}, T)$ curves approach a plateau as $T$ is lowered. This plateau occurs at values increasingly larger than the quiescent diffusion coefficient. Such an effect is a manifestation that the system shows shear-thinning behaviour. At high temperatures, a finite $\dot{\gamma}$ has only a small effect on the diffusion coefficient, and consequently all curves shown in the figure approach each other for small $1 / T$.

To study the effects of switching on shear flow suddenly, we consider a fixed temperature $T=0.14$, corresponding to the lowest temperature where we could equilibrate the quiescent system. This allows us to start from a well-defined reference state at $t=0$, and enables direct comparison with the theory. It also precludes aging effects arising from the unsheared state.

The macroscopic stress $\sigma(t)$ measured from the time of switching on steady shear is shown in Fig. 4 for three different shear rates. The kinetic contribution is negligible 


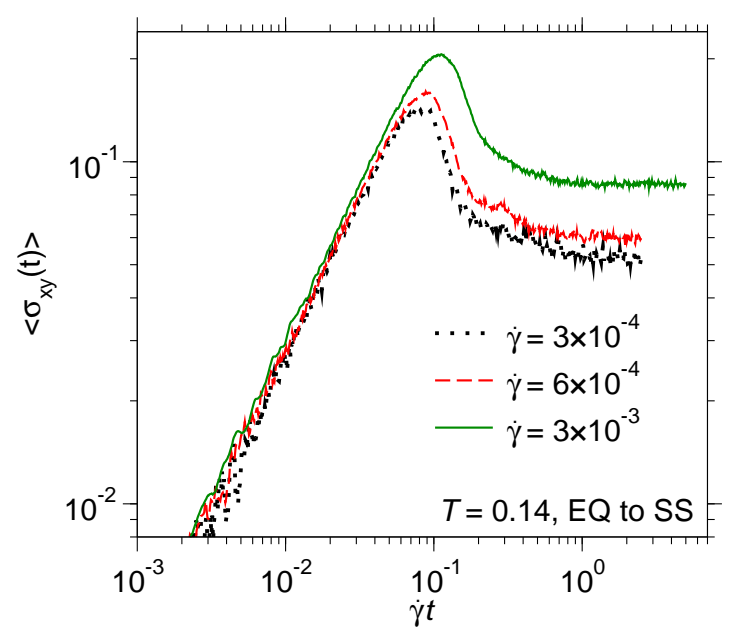

Figure 4. Stress-strain relation from the simulation at the temperature $T=0.14$ for three different shear rates, as indicated.

compared to the potential one, the one studied in the theory. All three curves are qualitatively similar, and show three regimes. At early times, corresponding to small strains $\gamma=\dot{\gamma} t \lesssim 0.1, \sigma(\gamma)$ increases almost linearly with $\gamma$. This is the regime of solid-like elastic response, and from the prefactor of this almost linear increase one can infer the elastic constant. It depends weakly on $\dot{\gamma}$. At large strains, $\gamma \gtrsim 1$, one finds on the other hand that $\sigma(t)$ approaches an asymptotic value attained in steady state, the dynamic shear stress $\sigma(\infty)$ resulting from keeping the system flowing at a fixed rate. This corresponds to the regime of plastic deformation. The long-time plateau increases with increasing $\dot{\gamma}$, indicating that faster flow induces higher internal stresses. The dependence of $\sigma(\infty)$ on shear rate is called 'flow curve'.

At strains in the intermediate range, $0.1 \lesssim \gamma \lesssim 1$, a marked overshoot is seen in $\sigma(\gamma)$ : the almost linear increase for small strains continues beyond the steady-state value of $\sigma$, so that a maximum $\sigma_{\max }>\sigma(\infty)$ results at roughly $10 \%$ strain in units of the particle diameters, slightly increasing with increasing shear rate. Only after this maximum does $\sigma(\gamma)$ decay towards its steady-state value. Similar features have been observed in experiment [11, 12, 13] and simulation [6, 16, 18, 19].

Since the simulation introduces shear flow only through the boundary conditions, the sudden switching on of shear does not induce a linear flow profile instantaneously throughout the simulation box. Rather, there exists some time scale over which the steady-state velocity profile builds up. Especially since we intend to compare the simulation results with MCT, where a fixed velocity profile is presumed to exist always, let us point out that none of the prominent features shown in Fig. 4 depend on this gradual self-adjustment of the profile. To this end, we determined the instantaneous velocity profiles, i.e., $v_{\mathrm{s}, x}(y)$, for the $\dot{\gamma}=3 \times 10^{-3}$ simulation at various times following shear startup. They have been obtained by averaging over a negligibly small time window and over the 250 independent simulation runs. As Fig. 5 shows, the profile for $t=0.6$ indeed still shows a pronounced ' $\mathrm{S}$ '-shaped form, as the LeesEdwards boundary conditions essentially introduce small sheared boundary zones to the unperturbed bulk. The propagation of these shear zones inwards is, however, fast. 


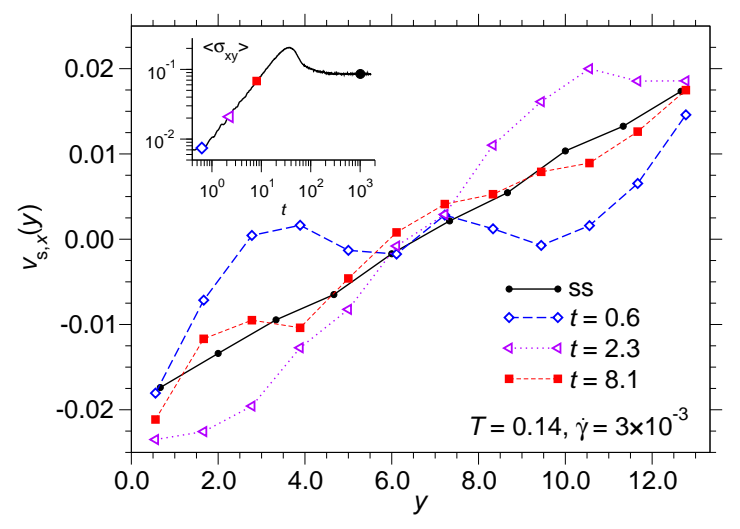

Figure 5. Evolution of the shear profile from equilibrium to steady state, as obtained from the simulation of the binary Yukawa mixture. Between the equilibrium and the steady state profiles are shown at the times $t=0.6, t=2.3$ and $t=8.1$. The applied shear rate is $\dot{\gamma}=3 \times 10^{-3}$. The temperature is $T=0.14$. The inset shows the stress as a function of time for $\dot{\gamma}=3 \times 10^{-3}$. The symbols in the inset indicate the times along the stress curve at which the shear profiles are shown.

Already around $t=8.1$, corresponding to a strain of $\gamma \approx 0.02$, one essentially recovers a linear velocity profile across the whole system within the error bars. This observation also holds for larger times (not shown). For comparison, also the profile obtained in the steady-state part of the simulation (averaged over a longer time interval and hence displaying smaller fluctuations) is shown in the figure. We do not find the formation of shear bands or similar localized features in the flow profile, even though the simulated equations of motion do not bias towards a linear profile by construction.

Comparing the strain at which the steady-state flow profile is first attained with the typical strains identified from the overshoot features in Fig. 4. we conclude that the latter are not connected to the peculiarities of whether the system is sheared only at the boundaries or forced to follow a linear profile mediated through the solvent immediately. This makes comparisons to MCT viable.

More information on the transient dynamics of the system can be obtained from two-time correlation functions, formed between two times $t_{\mathrm{w}}>0$ and $t>t_{\mathrm{w}}$. As a simple example, we show in Fig. 6 the mean-squared displacements (MSD) of B particles, for three different waiting times, $t_{\mathrm{w}}=0, t_{\mathrm{w}}=28.1$, and $t_{\mathrm{w}}$ sufficiently large so that the result as a function of $t-t_{\mathrm{w}}$ becomes $t_{\mathrm{w}}$-independent. For comparison, also the equilibrium MSD is shown. It agrees with the MSDs under shear for short times. Note that the solid lines correspond to the MSD evaluated in the gradient direction, while the dotted equilibrium and steady-state lines represent averages over both directions perpendicular to shear. Comparing steady-state and equilibrium, we recover for long times the shear-thinning effect discussed in connection with the diffusion coefficients, Fig. 3. The equilibrium curve shows a pronounced plateau where $\left\langle r^{2}\left(t, t_{\mathrm{w}}\right)\right\rangle \approx 0.013=r_{\text {plat }}^{2}$, indicating that particles are at intermediate times caged, with a localization length around $r^{1} \equiv r_{\text {plat }}^{2} / 6 \approx 0.02$. This is the usual glassy dynamics described by MCT in quiescent systems. The steady-state curve does not show such a pronounced plateau, due to the speed-up of the final relaxation.

As Fig. 6] shows, the sudden start-up of shear flow has a drastic effect on 

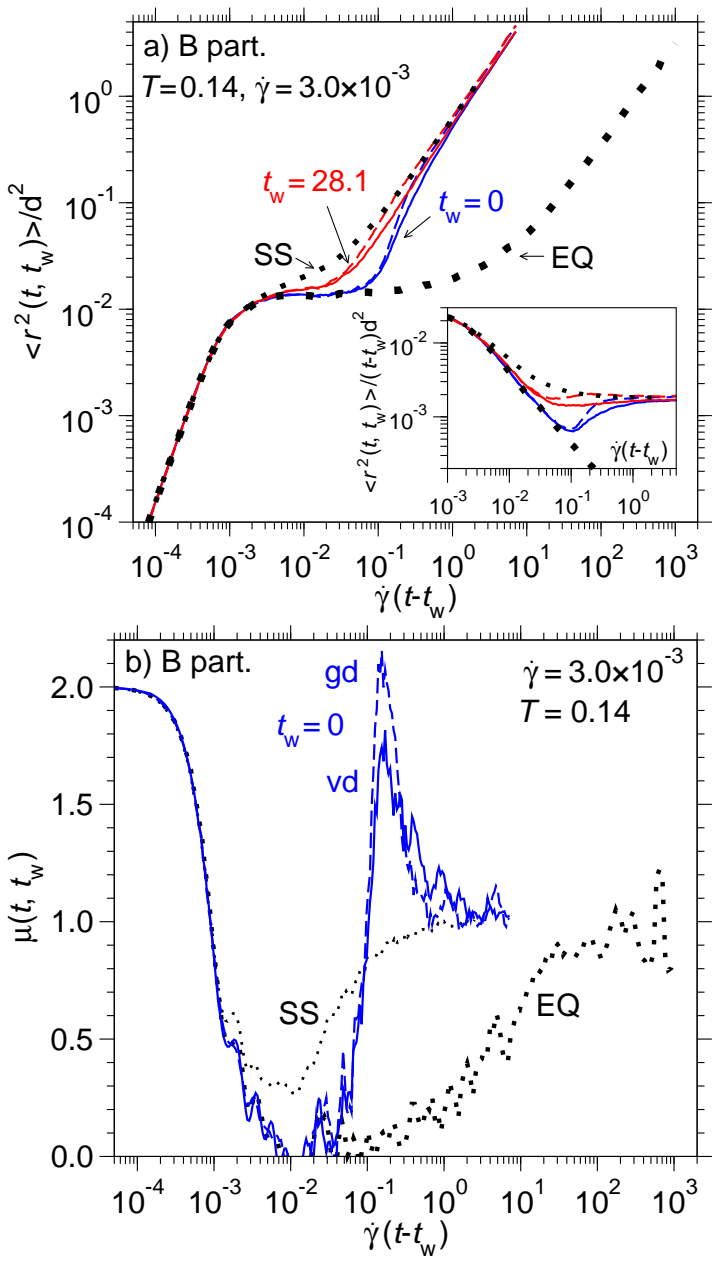

Figure 6. a) Log-log plot of the mean squared displacements of B particles at the temperature $T=0.14$ as a function of strain $\gamma \equiv \dot{\gamma} t$. Dashed and dotted lines correspond to equilibrium and steady state rescaled with $\dot{\gamma}=3 \times 10^{-3}$. The solid and dashed lines show the transition from equilibrium to steady state at this $\dot{\gamma}$ for the vorticity direction ( $z$, 'vd') and the gradient direction ( $y$, 'gd'), respectively. Data for the waiting times $t_{\mathrm{w}}=0$ and 28.1 are shown (from right to left). The dotted lines correspond to the mean squared displacements in the equilibrium (EQ) and in the steady state (SS), as indicated. In the inset, $\left\langle r^{2}\left(t, t_{\mathrm{W}}\right)\right\rangle /\left(t-t_{\mathrm{w}}\right)$ is plotted as a function of strain. b) Effective exponent $\mu(t)=d\left[\log \left\langle r^{2}\left(t, t_{\mathrm{w}}\right)\right\rangle\right] / d\left[\log \left(t-t_{\mathrm{w}}\right)\right]$ for some of the curves shown in a). 
the MSD measured immediately at the start-up time. While the curve follows the quiescent MSD for strains $\gamma \lesssim 0.1$, for larger time it suddenly starts to increase much more rapidly, and coincides with the steady-state MSD already for $\gamma \approx 1$, although $D(\dot{\gamma}) \gg D(\dot{\gamma}=0)$. This effect is seen in both directions perpendicular to the flow, albeit somewhat less pronounced in the vorticity direction, as demonstrated by the solid lines in Fig. 6. There is thus an intermediate time window where the MSD shows super-diffusive behaviour, i.e., grows faster than $t$. This is even more clearly seen when plotting $\left\langle r^{2}\left(t, t_{\mathrm{w}}\right)\right\rangle /\left(t-t_{\mathrm{w}}\right)$, as shown in the inset of Fig. 6. At long times, this curve would monotonically fall to $2 D$ in the quiescent system. A dip followed by an increase at intermediate times, as seen in our data, is the signature of superdiffusion.

The effect can be quantified more precisely through a logarithmic derivative, $\mu(t)=d\left[\log \left\langle r^{2}\left(t, t_{\mathrm{w}}\right)\right\rangle\right] / d\left[\log \left(t-t_{\mathrm{w}}\right)\right]$, which approaches $\mu=1$ for ordinary diffusion and $\mu=2$ for ballistic motion. The simulation result for $\mu(t)$ and $t_{\mathrm{w}}=0$ is shown both for the vorticity direction and the gradient direction in the lower panel of Fig. 6 At short times, $\mu(t \rightarrow 0) \rightarrow 2$ reveals the ballistic motion underlying the molecular dynamics simulation. At $t \approx 1, \mu(t) \approx 0$ is indicative of the equilibrium cage effect. In the $t_{\mathrm{w}}=0$ curve, it is truncated at $\dot{\gamma} t \approx 0.1$, and quickly $\mu(t)$ raises to about 2 in the gradient direction, and 1.8 in the vorticity direction, before finally settling to $\mu(t \rightarrow \infty) \rightarrow 1$ as expected. The simulation thus reveals an almost 'ballistic' regime in the MSD at intermediate times. Remarkably, this result remains unchanged if one performs the simulation with a much larger damping coefficient in the DPD thermostat, $\xi=1200$ [33]. This highlights that the regime of superdiffusion is likely a feature of sheared glassy dynamics and not of the short-time microscopic motion. It is worth noting that $\mu \leq 1$ holds for all times beyond the microscopic transient in the steady-state curve. This is what one expects from the regime of true 'structural relaxation', and what holds precisely in the Brownian equilibrium system, where all correlation functions are purely relaxing functions.

Figure 7 displays the non-Gaussian parameter $\alpha_{2}(t)$, as defined by Eq. (7), for equilibrium, steady state and waiting time $t_{\mathrm{w}}=0$ at $T=0.14$ and $\dot{\gamma}=3 \times 10^{-3}$. Also shown is the equilibrium $\alpha_{2}(t)$ at $T=0.18$ where $D(\dot{\gamma}=0)$ is approximately equal to $D\left(\dot{\gamma}=3 \times 10^{-3}\right)$ at $T=0.14$. In the steady state the amplitude of maximum in $\alpha_{2}(t)$ is about an order of magnitude smaller than that for the equilibrium at $T=0.14$ and also slightly smaller than that of the equilibrium curve at $T=0.18$. Thus the application of shear seems to reduce non-Gaussian effects at intermediate times. Similar to the behaviour of the MSD, the non-Gaussian parameter for waiting time $t_{\mathrm{w}}=0$ follows first the equilibrium curve. Then, at time $t \gtrsim 30$ the decay of $\alpha_{2}$ can be fitted to a power law, $\alpha_{2} \propto t^{-0.95}$.

Let us now compare in detail the findings from the simulation to the colloidal experiment. Figure 8 shows the mean-squared displacements obtained from confocal microscopy and particle tracking, taken in the vorticity direction. Here only a single shear rate is shown. Note in comparing with Fig. 6] that the relevant quantities characterizing the magnitude of shear differ between the two setups: while the experiment corresponds to a Weissenberg number $\mathrm{Pe} \approx 7\left(\mathrm{Pe}_{0} \approx 1\right)$, it is closer to $\mathrm{Pe} \approx 288$ in the simulated system. Apart from obvious differences due to this circumstance, the results are qualitatively similar. As the inset of Fig. 8 demonstrates for the experimental $\left\langle r^{2}\left(t, t_{\mathrm{w}}\right)\right\rangle /\left(t-t_{\mathrm{w}}\right)$, again a nonmonotonic dip is found at roughly $\gamma \approx 0.1$, identifying a corresponding super-diffusive regime in the MSD. Like in the simulation, this effect is most pronounced for $t_{\mathrm{w}}=0$ and then continuously weakens as the steady state is approached. 


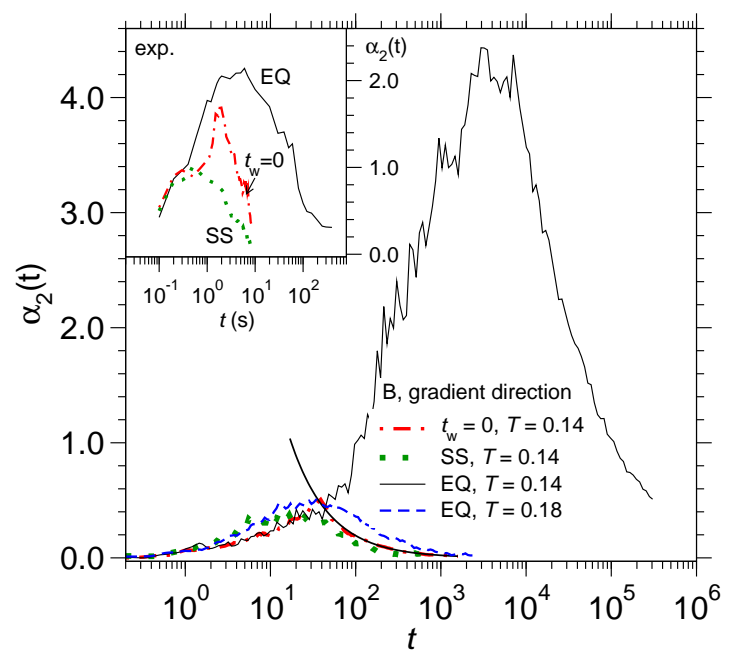

Figure 7. Non-Gaussian parameter $\alpha_{2}(t)$ for equilibrium, steady state and waiting time $t_{\mathrm{w}}=0$ at the temperature $T=0.14$. For the sheared case, the shear rate is $\dot{\gamma}=3 \times 10^{-3}$. For comparison also the equilibrium $\alpha_{2}(t)$ at $T=0.18$ is shown. $D(\dot{\gamma}=0)$ at this temperature is approximately equal to $D\left(\dot{\gamma}=3 \times 10^{-3}\right)$ at $T=0.14$. The bold solid line is a fit to the $t_{\mathrm{w}}=0$ curve with the indicated power law $\alpha_{2} \propto t^{-0.95}$. The inset shows experimental results for $\alpha_{2}(t)$. Here, the shear rate is $\dot{\gamma}=4.5 \times 10^{-2} \mathrm{~s}^{-1}$.

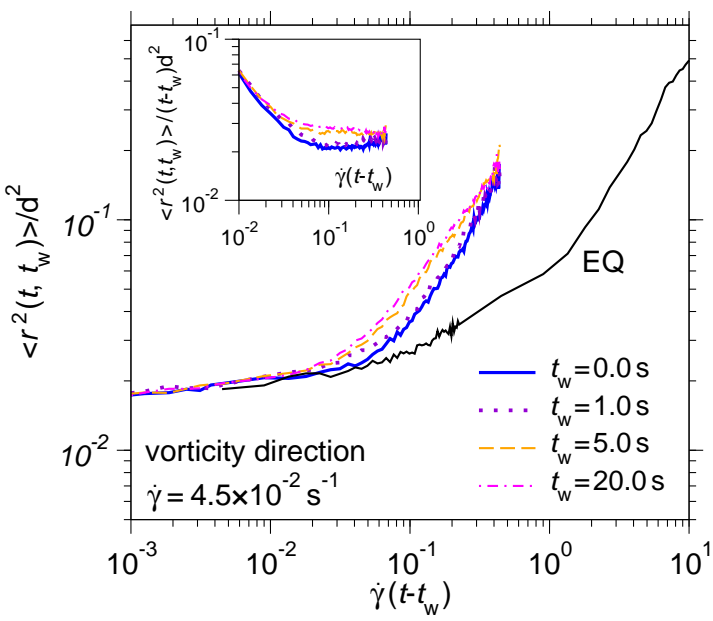

Figure 8. Log-log plot of the experimental mean squared displacement in vorticity direction as a function of strain $\dot{\gamma} t$ for different waiting times, as indicated. The inset shows $\left\langle r^{2}\left(t, t_{\mathrm{w}}\right)\right\rangle /\left(t-t_{\mathrm{w}}\right)$ as a function of $\dot{\gamma} t$. 


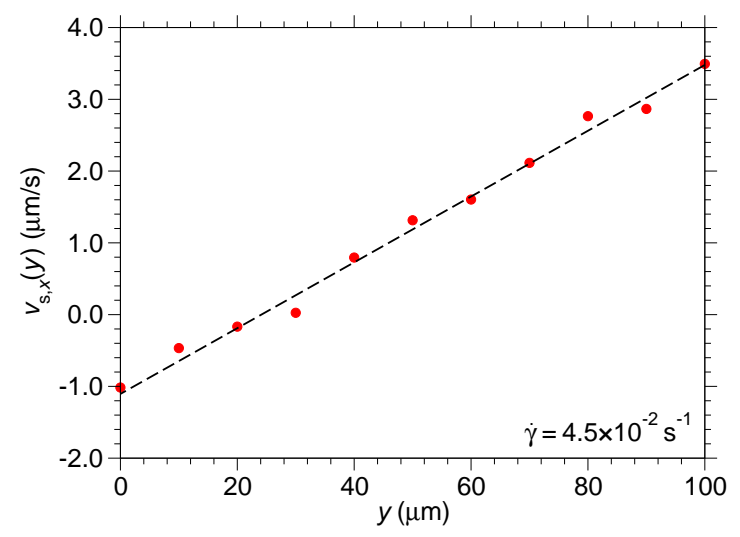

Figure 9. Steady state shear profile, as obtained from the experiment (dots). Dashed line: linear fit providing $\dot{\gamma}=0.045 \mathrm{~s}^{-1}$.

A quantitative difference is found when comparing the extension and strength of the super-diffusive window in the MSD. In the simulation, the crossover in the $t_{\mathrm{w}}=0$ curve from the equilibrium to the steady-state limiting cases is much more rapid. Likewise, the experiments show the first increase of the transient MSD beyond the equilibrium curve for slightly smaller strains, $\gamma \approx 0.05$, than the $\gamma \approx 0.1$ found in the simulation.

Also the non-Gaussian parameters have been determined in the experiment. They are shown in the inset of Fig. 7. One can infer that also this quantity behaves qualitatively as in the simulation.

Again it is important to stress that the effects discussed here are not consequences of shear localization or, more generally, developments of non-linear flow profiles. This has also been checked for the experiment. The velocity profile in steady state is almost perfectly linear, as Fig. 9 demonstrates. This confirms that also in the experimental setup, a homogeneous constant-shear steady state is approached, without obvious shear-localization features or wall effects.

Having established the close connection between simulation and experiment, let us now try to rationalize these findings with MCT. The quantity $\sigma(t)$ is easily accessed in the mode-coupling framework and thus forms a convenient starting point for the analysis. In order to provide a meaningful comparison with simulation results, we have to first map the relevant quantities between the theoretical hard-sphere model and the binary Yukawa mixture employed in the simulations. Since we are dealing with slow structural dynamics, it is natural to fix the final relaxation time $\tau$ first for equilibrium and then to consider the effect of shear identifying the Péclet and Weissenberg numbers. The only free parameter in the quiescent hard-sphere model is the packing fraction, adjusted in the following to $\Delta \phi=\phi-\phi_{c}=-1.16 \times 10^{-3}$ from the requirement that MCT describes the equilibrium MSD found in the simulation for long times (see below). Next, the shear rate appropriate for use in the theory may be determined. We find $\mathrm{Pe}=183$ (corresponding to $\dot{\gamma}=5.5 \times 10^{-3} D_{0} / d^{2}$ ) to lead to the same $D(\dot{\gamma}) / D(\dot{\gamma}=0)$ as it arises from the simulation at $\mathrm{Pe} \approx 288$.

In Fig. 10 we show the resulting MCT-calculated stress $\sigma(t)$ as a function of accumulated strain $\dot{\gamma} t$ for the three shear rates considered in the simulation. 


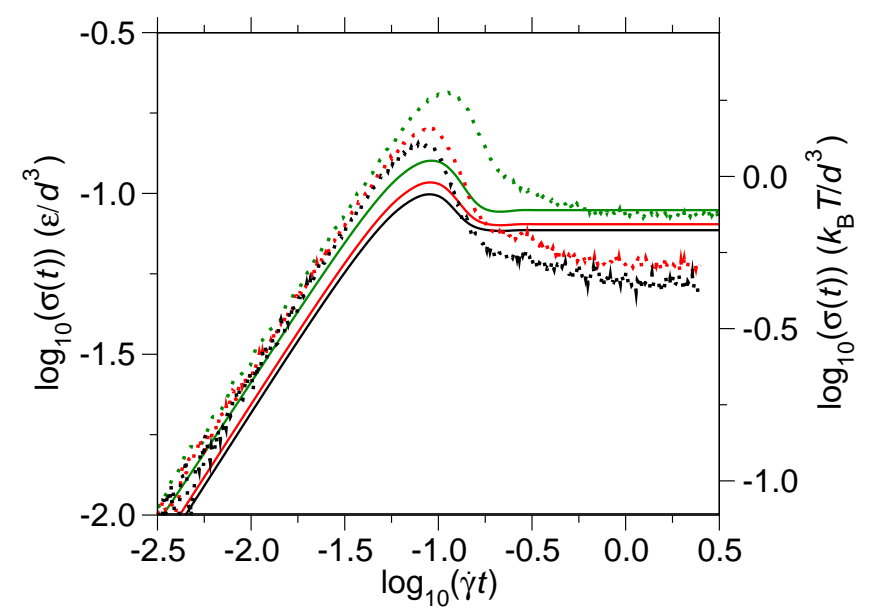

Figure 10. Comparison of the stress from simulation (dots) and theory (solid lines) for the shear rates considered in simulation, $\dot{\gamma}=3 \times 10^{-3}$ (green), $\dot{\gamma}=6 \times 10^{-4}$ (red) and $\dot{\gamma}=3 \times 10^{-4}$ (black). The relation between simulation energy units $\left(E_{\mathrm{md}}\right)$ and theory is $E_{\mathrm{md}}=8.8 k_{B} T$. The theoretical shear rates (in units of $d^{2} / D_{0}$ ) obtained from fitting the MSD at the highest shear rates of the simulation (corresponding to a theoretical value $\mathrm{Pe}=183$ ) are $\dot{\gamma}=5.5 \times 10^{-3}$, $\dot{\gamma}=1.1 \times 10^{-3}$ and $\dot{\gamma}=5.5 \times 10^{-4}$, respectively.

Theoretical results are shown in hard-sphere units $\left(k_{B} T / d^{3}\right)$ whereas simulation results are expressed in units of $E_{\mathrm{md}}=\epsilon / d^{3}$. The two axes have been adjusted in order to match the stress attained in steady state, fixing a relation of $E_{\mathrm{md}} \approx 8.8 k_{B} T$ between the kinetic energies of the different systems for the largest shear rate considered in the simulation. This fitted value of $T$ is surprisingly close to the actual value in the simulation, indicating that the theory captures the stress magnitude well. As Fig. 10 shows, this also provides a reasonable description of the initial small-strain stress, reflecting the elastic constant of the system. More importantly, the theory qualitatively reproduces the stress overshoot found in the simulation. That the peak occurs at strains of roughly $\gamma \approx 0.1$, in accord with simulation, is a consequence of our choice for the parameter $\gamma_{c}$ in the isotropic approximation to the advected wavevector. The magnitude of the overshoot is significantly underestimated, which might be due to the MCT approximation per se, but may also be a result of the additional isotropic approximation underlying our calculations. In the decay of $\sigma(t)$ after its maximum, the simulation data show a rather slow decay for $\log _{10} \gamma \gtrsim 0.75$, which is also not captured in MCT.

The theory quite naturally relates this stress overshoot to a peculiar feature of the dynamic shear modulus $G(t)$. In Fig. 11 this modulus is shown both in the quiescent case and for the shear rates used in Fig. 10. The equilibrium function shows the two-step relaxation pattern familiar from other correlation functions close to the glass transition. The non-linear shear thinning is clearly seen as a speeding up of the final relaxation time in $G(t)$ from the equilibrium case $\left(\tau \approx 10^{3}\right)$ to that of steady-shear, for which the characteristic time scale is $\dot{\gamma}^{-1}$. A plateau at times around $t \approx 1$ is still seen as a remnant of the cage effect. Note that only much closer to the glass transition would a well-established constant plateau develop, defining the solid shear modulus $G_{\infty}$. For the parameters chosen here, the modulus $G(t)$ still shows a finite slope in 


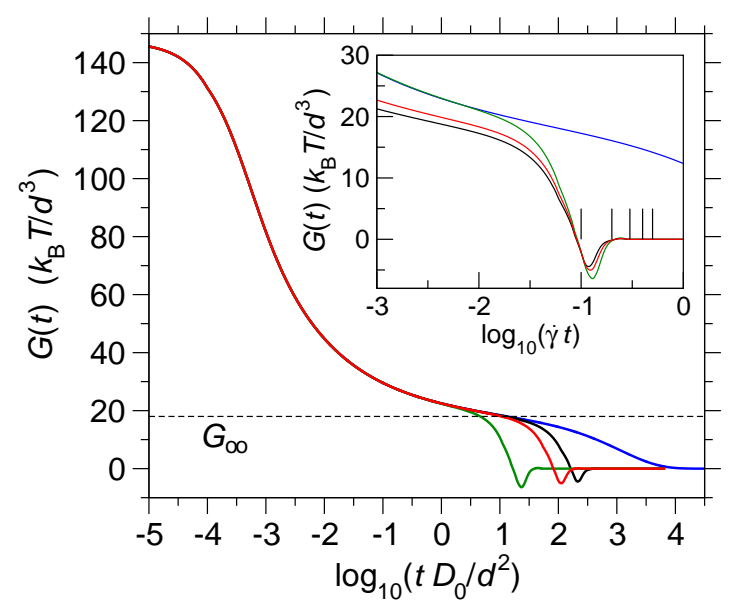

Figure 11. Generalized shear modulus from mode-coupling theory, calculated using $\phi-\phi_{c}=-1.16 \times 10^{-3}$, for $\dot{\gamma}=0$ (rightmost curve) and $\dot{\gamma}=5.5 \times 10^{-3}$, $1.1 \times 10^{-3}$, and $5.5 \times 10^{-4}$ (left to right), corresponding to curves in figure 10 The stress overshoot in Fig. 10 is related to the negative undershoot in $G(t)$ at long times. The plateau value which would develop closer to the glass transition is indicated by the broken line. The inset shows the moduli as a function of strain, where the vertical bars indicate the strains considered in Fig. 12

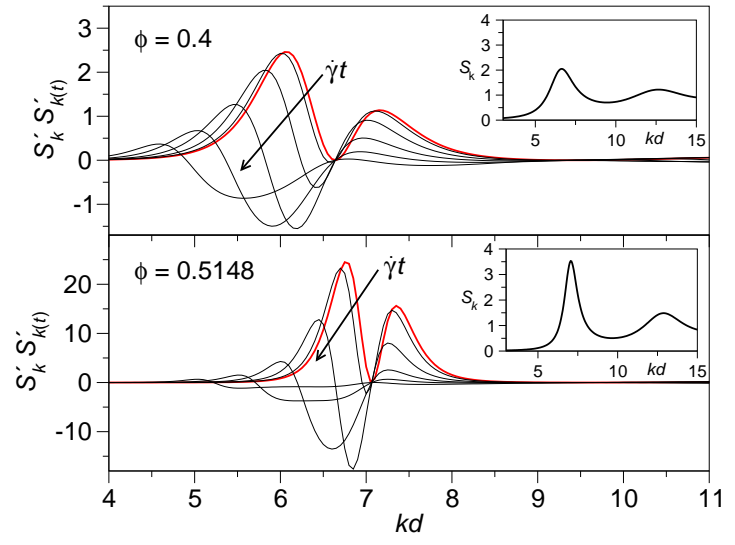

Figure 12. The product $S_{k}^{\prime} S_{k(t)}^{\prime}$ which enters the stress vertex and which leads to negative values of the modulus $G(t)$ at long times. The uppermost panel and inset show $S_{k}^{\prime} S_{k(t)}^{\prime}$ and $S_{k}$, respectively, for a packing fraction $\phi=0.4$. Curves are for strain values $\dot{\gamma} t=0,0.1,0.2,0.3,0.4$ and 0.5 (where the arrow indicates the direction of increase). The lower panel shows the same quantities for a packing fraction of $\phi=0.5148$, corresponding to the packing fraction used in our comparison with simulation. The five values of the strain considered are marked in Fig. 11

the plateau regime. Considering that $\sigma(t)$ is the time-integral over $G(t)$ leads to the conclusion that the 'elastic' small-strain regime visible in Figs. 4 and 10 is not truly linear in $\gamma$. Indeed, the simulation data could be fitted with a power law $\sigma \propto \gamma^{x}$ with some effective exponent $x<1$ in this time window. 
The transient shear modulus in addition shows a characteristic negative dip for strains around $\dot{\gamma} t \approx 0.1$, which for the highest shear rate corresponds to $t D_{0} / d^{2} \approx 20$. From the integral for the stress, Eq. (11), it is apparent that an overshoot in $\sigma(t)$ is the result of such a negative portion in $G(t)$ for long times. Recall that $G(t)$ is a stress-stress autocorrelation function, so that this negative dip may be interpreted as a 'backscattering' of stresses. It arises from the final stage of the relaxation, commonly referred to as the $\alpha$ process, which is attributed to the breaking up of nearest-neighbour cages. As a result MCT does not correlate the stress overshoot to peculiar inhomogeneities, such as localized shear events.

Within our MCT-based approach negative values of the modulus occur as a result of the factor $S_{k}^{\prime} S_{k(t)}^{\prime}$ appearing in Eq.(12). The derivative $S_{k}^{\prime}$ oscillates about zero as a function of $k$ and attains maximal positive and negative values in the vicinity of the first peak in $S_{k}$. As the strain is increased the resulting de-phasing of the two factors $S_{k}^{\prime}$ and $S_{k(t)}^{\prime}$ leads to negative regions in the product $S_{k}^{\prime} S_{k(t)}^{\prime}$. This behaviour is shown in more detail in Fig 12 for two values of the packing fraction $\phi$, where the structure factors are taken from the analytic Percus-Yevick theory. Comparing the curves for the two densities it is apparent that the first peak in $S_{k}$ is considerably narrower and steeper for $\phi=0.5148$ than for $\phi=0.4$, leading to larger values of $S_{k}^{\prime}$ in this region. In each case the product $S_{k}^{\prime} S_{k(t)}^{\prime}$ becomes increasingly negative as the strain is increased from $\dot{\gamma} t=0.1$ to $\dot{\gamma} t=0.2$. At longer times, the decay of the correlators in Eq. (12) suppresses the negative vertex. The inset to Fig. 11] shows the modulus $G(t)$ as a function of strain. For strains in the range 0.1 to 0.25 the modulus attains its most negative values. Comparison of the two panels in Fig. 12 shows that for $\phi=0.5148$ the negative region in $S_{k}^{\prime} S_{k(t)}^{\prime}$ is much more developed at $\dot{\gamma} t=0.2$, relative to $\phi=0.4$. This reflects the differences in the first peak of the structure factor at the two densities and suggests that systems with a steeper and narrower first peak in $S_{k}$ will possess a more pronounced overshoot within the present MCT-based approach. Structure factors with a steeper and narrower first peak are known to occur in two-dimensional systems [50].

We have now established two features of the transient dynamics immediately following the instantaneous switch-on of shear, both of which arise at strains of about $10 \%$ of the particle diameter. The first of these, $\sigma(t)$ (equivalently $G(t)$ ), concerns the collective dynamics, while the other, $\left\langle r^{2}\left(t, t_{\mathrm{w}}\right)\right\rangle$, concerns the single-particle dynamics of a tracer particle. It is intriguing to see in which way these conceptually different pieces of information might be related and to connect the macroscopic rheological information contained in $\sigma(t)$ with the microscopic particle motion. We consider in more detail the transient MSD, $\delta r^{2}(t)=\left\langle r^{2}\left(t, t_{\mathrm{w}}=0\right)\right\rangle$. An idea that has proven particularly successful in the quiescent system is that of a generalized Stokes-Einstein (GSE) relation, proposed by Mason and Weitz as a correspondence between macroand micro-rheology [51]. Within this approach the diffusion coefficient $D$ is related to the viscosity $\eta$ by

$$
D \approx \frac{k_{B} T}{3 \pi d \alpha \eta}=\frac{k_{B} T}{3 \pi d \alpha \int_{0}^{\infty} d s G(s)} .
$$

In the context of quiescent MCT, this relation exemplifies the coupling of the $\alpha$ relaxation times [52], and even quantitatively works surprisingly well for the quiescent hard-sphere system [49]. Quantitatively accurate results can be obtained if one allows for a small correction to the geometrical prefactor $3 \pi d$ appearing in the original StokesEinstein relation, $\alpha \neq 1$. 


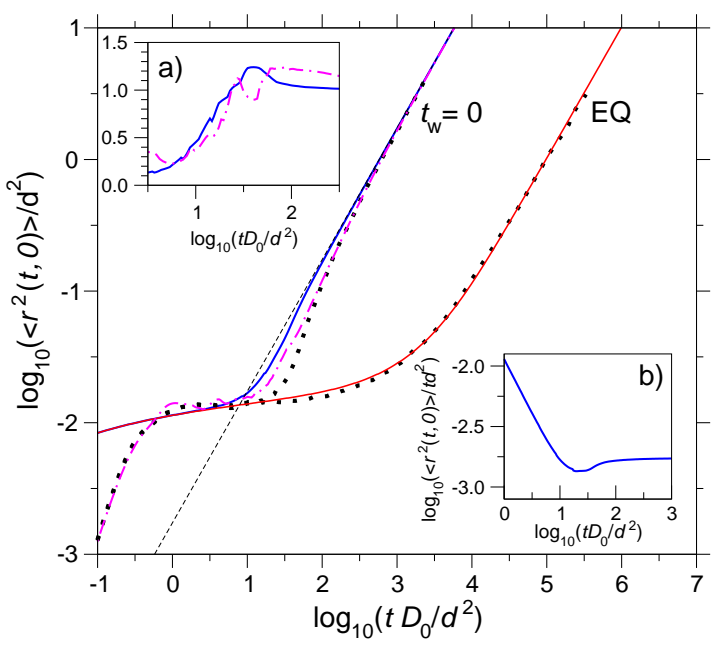

Figure 13. Comparison of the mean-square displacements from simulation (vorticity direction, dots) and theory (solid lines), for equilibrium (EQ) and for the highest shear rate considered in the simulations (waiting time $t_{\mathrm{w}}=0$ ). A superdiffusive regime is apparent in the theoretical MSD for $t_{\mathrm{w}}=0$, establishing the connection to the stress overshoot in Fig. 10. Inset (a) shows the effective exponent from the theory for $t_{\mathrm{w}}=0$ obtained from the logarithmic derivative. The peak is at a value of $\sim 1.2$. Inset (b) shows the MSD divided by $t$. Dash-dotted lines correspond to the MSD calculated from the simulated $\sigma(t)$ at $\dot{\gamma}=3 \times 10^{-3}$ and the GSE relation (see text). The dashed line indicates the linear diffusive behavior in steady state.

In addition to the correlator $\Phi_{k}(t), \mathrm{MCT}$ also yields equations of motion for the tagged-particle correlation functions, which yield an equation for the MSD in the $q \rightarrow 0$ limit. Taking the form of the equation of motion for $\delta r^{2}(t)$ from the quiescent theory (ignoring any generalizations that come about due to the anisotropic flow geometry), we can write

$$
\delta r^{2}(t)+\frac{D_{0} d}{k_{B} T} \int_{0}^{t} d t^{\prime} m^{(s)}\left(t-t^{\prime}\right) \delta r^{2}\left(t^{\prime}\right)=6 D_{0} t,
$$

where $m^{(s)}(t)$ is the tagged-particle memory kernel evaluated as $q \rightarrow 0$. An analysis of the above equation at large times immediately gives an expression for the diffusion coefficient in terms of the memory kernel,

$$
D=\frac{D_{0}}{1+D_{0} \int_{0}^{\infty} d s m^{(s)}(s)} \approx \frac{1}{\int_{0}^{\infty} d s m^{(s)}(s)} .
$$

Comparison of (17) with (15) clearly establishes a relationship between the time integrals of $m^{(s)}(t)$ and $G(t)$. However, the connection is deeper than simple equality of the integrated quantities. Studies performed using the quiescent mode coupling theory have established that the MCT approximation to $G(t)$ is in excellent agreement (up to a constant prefactor) with the MCT approximation to $m^{(s)}(t)$ for the hard sphere system [49]. In this work we make the assumption that this correspondence holds also in the non-linear regime for which $G(t) \equiv G(t, \dot{\gamma})$. Given this assumption we obtain a direct correspondence between the MSD and the derivative of the shear stress after 
startup, $(d / d t) \sigma(t) \propto G(t)$,

$$
m^{(s)}(t) \approx\left(\frac{d}{k_{B} T}\right) 3 \pi \alpha G(t)=\left(\frac{d}{k_{B} T}\right) \frac{3 \pi \alpha}{\dot{\gamma}} \frac{d}{d t} \sigma(t) .
$$

We now consider application of the GSE approximation (18) to the calculation of the mean-squared displacement using (16). In Fig. 13 we compare the $t_{\mathrm{w}}=0$ curves obtained from simulation and theory for the largest shear rate considered in the simulation. Also shown is the equilibrium curve that has been used to adjust $\Delta \phi$ in fitting the equilibrium simulation result. At short times differences arise due to the different microscopic dynamics, as expected, but for the long-times relevant to our discussion, the fit is convincing. The MCT-GSE theory displays a superdiffusive regime at intermediate times, qualitatively reproducing the phenomenology of the simulation. In order to quantify this superdiffusivity, we show in inset (a) the logarithmic derivative of the MSD, yielding an effective exponent for $\delta r^{2}(t)$. While in the simulation this quantity grows up to 2 (reflecting ballistic motion) in the intermediate regime, our MCT calculation produces effective exponents up to roughly 1.2. This situation is much closer to the results of our colloidal experiments.

Note that from (17), a super-diffusive regime in the MSD can only arise if $m^{(s)}(t)$ takes on negative values. By virtue of (18), the presence of a super-diffusive regime is therefore intimately connected to the stress overshoot and the corresponding overrelaxation feature found in $G(t)$. It is therefore tempting to assume that the more pronounced super-diffusive regime found in the simulations relative to the theory is a consequence of the correspondingly stronger stress overshoot (see Fig. 10). In order to investigate this connection further we have directly calculated the meansquared displacement from the simulation curve for $\sigma(t)$, using (18) to obtain the memory function $m^{(s)}(t)$. In this calculation, the Newtonian-dynamics equivalent of Eq. (16) was used with a prefactor $\alpha=0.833$ (accounting for the numerical uncertainty that arises from the neccessary smoothening of the derivative of the simulated $\sigma(t)$ ). The resulting MSD is shown in Fig. 13 (dash-dotted lines). For times longer than $t D_{0} / d^{2} \approx 100$ the MSD obtained in this fashion closely follows the directly simulated quantity. However, for shorter times the agreement is less satisfactory and significant deviations are visible. The logarithmic derivative shown in inset of Fig. 13] confirms this. As a result of these findings we conclude that the approximation (18) remains qualitatively reliable in the non linear regime but that it fails quantitatively in the super-diffusive time window where the stress overshoot is strongly pronounced.

Finally, we consider application of the GSE-MCT relation to fitting the experimental data for the MSD, the results of which can be seen in Fig. 14. We follow the same procedure as when fitting the simulation data and consider first the equilibrium MSD. By adjusting $\Delta \phi$ we fix the ratio of final to microscopic relaxation time and find a value $\Delta \phi=-1.1 \times 10^{-2}$ reproduces the experimental results to a satisfactory level, using also a value $\alpha=0.95$ little different from unity. We next increase the shear rate until the long time diffusion matches that of the simulation. This is achieved for $\mathrm{Pe}=16$ (corresponding to $\dot{\gamma}=5 \times 10^{-2} D_{0} / d^{2}$ ). We find a superdiffusive regime in qualitative agreement with the experiment at $\mathrm{Pe}=7$, but which is somewhat underestimated in magnitude. This lends support to our argument that the approximation (18) is less reliable in the super-diffusive regime than in equilibrium. In the inset we show the MSD divided by $t$ which confirms the relatively weaker superdiffusion. 


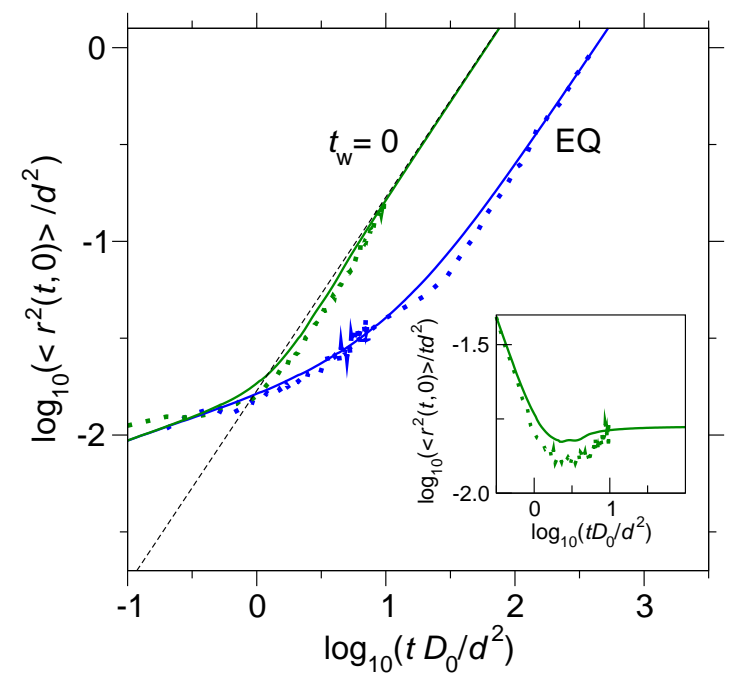

Figure 14. Theoretical fits to the experimental mean-square displacement (dotted) for equilibrium and $t_{\mathrm{w}}=0$ (solid lines) using $\alpha=0.95$ (see text). The inset shows the MSD divided by $t$ and highlights the super-diffusive regime.

\section{Conclusions}

We have studied the transient dynamics of a glassforming system that is subjected to the sudden commence of steady shear flow. Results from computer simulation, confocal-microscopy experiment, and mode-coupling theory give a consistent picture that reveals peculiar dynamical features around the time where the system reaches $10 \%$ strain for the first time. In particular, the mean-squared displacements in both the vorticity and the gradient direction show super-diffusive behaviour at intermediate times, interpolating between the equilibrium curve that is followed for $\gamma \lesssim 0.1$ and the faster steady-state curve that is followed for $\gamma \gtrsim 1$. The super-diffusive behaviour is more pronounced in the (ballistic) simulation, giving rise to effective exponents of 2 , and less pronounced in the colloidal experiment.

Again at strains of $\gamma \approx 0.1$, a well-recognized feature in the collective dynamics is found, visible in the building up of the shear stress as a 'stress overshoot' (a maximum in $\sigma(t)$ followed by a decrease towards a smaller steady-state value at later times). While we cannot propose a simple picture of microscopic motion behind this stress overshoot, we are able within MCT to directly relate it to a 'stress overrelaxation', visible in the corresponding stress-stress autocorrelation function in the late $\alpha$-relaxation regime. It can thus be attributed to the peculiar way in which cages break up, and MCT predicts its specific shape and magnitude to be systemdependent. We have demonstrated that $\mathrm{MCT}$ in this way qualitatively describes the stress overshoot seen in the simulation, albeit quantitatively underestimated.

A generalized Stokes-Einstein relation can be used to relate the above two features of the transient collective and single-particle dynamics. We have used MCT in combination with this close link between micro- and macro-rheology in order to demonstrate that the theory-predicted stress overrelaxation quite naturally leads to a super-diffusive regime in the mean-squared displacement. Again, its magnitude is less 
pronounced than in the simulation, but compares reasonably well with experiment, so that the remaining differences can be attributed to the microscopic difference in the systems, the isotropic nature of our approximations, and violations of the generalized Stokes-Einstein relation.

\section{Acknowledgments}

We thank K. Binder for useful discussions. We thank R. Besseling for providing routines for calculation of the velocity profile, A. Schofield for providing PMMA particles and D. Vobis for help with the design and construction of the shear cell. We thank SFB TR6 for support. Th. V. thanks for funding through the Helmholtz-Forschungsgemeinschaft (Impuls- und Vernetzungsfonds, VH-NG-406). We acknowledge a substantial grant of computer time at the Jülich multiprocessor system (JUMP) of the John von Neumann Institute for Computing (NIC).

\section{References}

[1] Larson R G 1999 The Structure and Rheology of Complex Fluids (Oxford, Oxford University Press)

[2] Yamamoto R and Onuki A 1998 Phys. Rev. E 58 3515-3529

[3] Strating P 1999 Phys. Rev. E 592175

[4] Berthier L and Barrat J L 2002 Phys. Rev. Lett. 89095702

[5] Varnik F, Bocquet L, Barrat J L and Berthier L 2003 Phys. Rev. Lett. 90095702

[6] Varnik F, Bocquet L and Barrat J L 2004 J. Chem. Phys. 1202788

[7] Varnik F and Henrich O 2006 Phys. Rev. B 73174209

[8] Varnik F 2006 J. Chem. Phys. 125164514

[9] McPhie M G, Daivis P J and Snook I K 2006 Phys. Rev. E 74031201

[10] Besseling R, Weeks E R, Schofield A B and Poon W C K 2007 Phys. Rev. Lett. 99028301

[11] Osaki K, Inoue T and Isomura T 2000 J. Polym. Sci. B 381917

[12] Islam M T and Archer L A 2001 J. Polym. Sci. B 392275

[13] Letwimolnun W, Vergnes B, Ausias G and Carreau P 2007 J. Non-Newtonian Fluid Mech. 141167

[14] van Aken B, de Hey P and Sietsma J 2000 Mater. Sci. Eng. A 278247

[15] Johnson W L, Lu J and Demetriou M D 2002 Intermetallics 101039

[16] Heyes D M 1986 J. Chem. Phys. 85997

[17] Utz M, Debenedetti P G and Stillinger F H 1999 Phys. Rev. Lett. 841471

[18] Rottler J and Robbins M O 2003 Phys. Rev. E 68011507

[19] Tanguy A, Leonforte F and Barrat J L 2006 Eur. Phys. J. E 20355

[20] Fuchs M and Cates M E 2002 Phys. Rev. Lett. 89248304

[21] Fuchs M and Cates M E 2005 J. Phys. Cond. Matt. 17 S1681

[22] Fuchs M and Cates M E 2003 Faraday Discussions 123267

[23] Brader J M, Voigtmann Th, Cates M E and Fuchs M 2007 Phys. Rev. Lett. 98 058301 
[24] Brader J M, Cates M E and Fuchs M To be published

[25] Miyazaki K, Reichman D R and Yamamoto R 2004 Phys. Rev. E 70011501

[26] Miyazaki K, Wyss H M, Weitz D A and Reichman D R 2007 Europhys. Lett. 75 915

[27] Wyss H M, Miyazaki K, Mattsson J, Hu Z, Reichman D R and Weitz D A 2007 Phys. Rev. Lett. 98238303

[28] Szamel G 2004 Phys. Rev. Lett. 93178301

[29] Lee A W and Edwards S F 1972 J. Phys. C: Solid State Phys. 51921

[30] Evans D J and Morriss G P 1990 Statistical Mechanics of Non-Equilibrium Liquids (London, Academic Press)

[31] Soddemann T, Dünweg B and Kremer K 2003 Phys. Rev. E 68046702

[32] Espanol P and Warren P 1995 Europhys. Lett. 30191

[33] Zausch J and Horbach J To be published

[34] Peters E A J F 2004 Europhys. Lett. 66311

[35] Nijboer B R A and Rahman A 1966 Physica 32415

[36] Boon J P and Yip S 1991 Molecular Hydrodynamics (New York, Dover)

[37] Yethiray A and van Blaaderen A 2003 Nature 421513

[38] Jenkins M 2005 Ph.D. thesis University of Edinburgh

[39] Jenkins M and Egelhaaf S 2008 Adv. Colloid Interface Sci. 13665

[40] Pusey P and van Megen W 1984 J. Chem. Phys. 803513

[41] Ackerson B and Pusey P 1988 Phys. Rev. Lett. 611033

[42] Petekidis G, Pusey P N, Moussaid A, Egelhaaf S and Poon W C K 2002 Physica A 306334

[43] Petekidis G, Moussaid A and Pusey P 2002 Phys. Rev. E 66051402

[44] Smith P, Petekidis G, Egelhaaf S and Poon W 2007 Phys. Rev. E 76041402

[45] Smith P 2004 Ph.D. thesis University of Edinburgh

[46] Crocker J and Grier D 1996 J. Colloid Interface Sci. 179298

[47] Isa L, Besseling R, Weeks E and Poon W 2006 J. Phys.: Conf. Ser. 40124

[48] Dhont J K G 1996 An Introduction to the Dynamics of Colloids (Amsterdam, Elsevier)

[49] Fuchs M and Mayr M R 1999 Phys. Rev. E 605742

[50] Bayer M, Brader J M, Ebert F, Lange E, Fuchs M, Maret G, Schilling R, Sperl M and Wittmer J 2007 Phys.Rev.E 76011508

[51] Mason T G and Weitz D A 1995 Phys. Rev. Lett. 741250

[52] Götze W and Sjögren L 1992 Rep. Prog. Phys. 55241 